1 Hacettepe Journal of Mathematics and Statistics

$\bigcap$ Volume 47 (4) (2018), 889-896

\title{
Statistical convergence of sequences of sets in hyperspaces
}

\author{
Sevda Sağıroğlu*† and Mehmet Ünver*
}

\begin{abstract}
The concept of statistical convergence in an arbitrary topological space is nothing new, it is actually a self-evident concept that comes through the structure of that space. In this paper, by considering the well known topologies on hyperspaces, we investigate the characterizations of statistical convergence of sequences of sets in the realm of these structures.
\end{abstract}

Keywords: Hyperspaces, weak topologies, statistical convergence.

Mathematics Subject Classification (2010): 54A20, 54B20, 40A35

Received : 07.06.2016 Accepted : 28.09.2016 Doi : 10.15672/HJMS.2017.482

\section{Introduction}

Hyperspace topologies and related concepts of convergence of sequences of sets have been considered since 1960 's. The theory was initially appeared with the work of Wijsman [28] and developed by Mosco [21], Wets [27], Attouch [1] and Beer [2],[3],[4]. If a sequence of sets does not converge then it might be benefical to use statistical convergence. Statistical convergence is one of the main concepts of the summability theory that can be introduced in an arbitrary topological space without the requirement of a lineer structure or at least a group structure on that space, so it is natural to consider statistical convergence of sequences of sets in the realm of hyperspaces. Maddox [18] studied statistical convergence in locally convex spaces, Maio and Kočinac [19] have considered it in topological spaces and there are some papers studying statistical convergence in hyperspaces [15], [22], [23], [25]. We add to all these the facts that are direct results of the topological view.

*Ankara University, Faculty of Science, Department of Mathematics, Tandoğan, Ankara TURKEY

Email : ssagir@science.ankara.edu.tr

${ }^{\dagger}$ Corresponding Author.

$\ddagger$ Ankara University, Faculty of Science, Department of Mathematics, Tandoğan, Ankara TURKEY

Email : munver@ankara.edu.tr 
An open set in a hyperspace $X$ is a family of closed subsets of the underlying topological or metric structure on $X$. As usual we will denote the nonempty closed subsets of $X$ by $C L(X)$ and the closed subsets of $X$ including the empty set by $2^{X}$. Most of the hyperspace topologies on closed subsets of a Hausdorff space $X$ are usually defined by subbases consisting of members in the following sense:

$$
A^{+}:=\{B \in C L(X) \mid B \subset A\} \text { and } A^{-}:=\{B \in C L(X) \mid A \cap B \neq \emptyset\}
$$

for an arbitrary subset $A$ in $X$.

In the case of a metric space $(X, d)$, by $S_{\alpha}[x]$ (resp. $\left.\bar{S}_{\alpha}[x]\right)$ we denote the open (resp. closed) ball with center $x$ and radious $\alpha$. Similarly for a subset $A$ of $X$ let

$$
S_{\alpha}[A]:=\{x \mid d(x, A)<\alpha\}, \bar{S}_{\alpha}[A]:=\{x \mid d(x, A) \leq \alpha\}
$$

and

$$
A^{++}:=\left\{B \in C L(X) \mid \exists \varepsilon>0 \ni S_{\varepsilon}[B] \subset A\right\} .
$$

Also, we will require the following well known set valued functions;

$$
e_{d}(A, B):=\inf \left\{\varepsilon>0 \mid S_{\varepsilon}[B] \supset A\right\}
$$

and

$$
D_{d}(A, B):=\inf \left\{\varepsilon>0 \mid A \cap S_{\varepsilon}[B] \neq \emptyset\right\}
$$

which are called excess and gap functions, respectively.

Given $\Delta \subset C L(X)$, the hit and miss topology $\tau(\Delta)$ is defined as the topology having as a subbase of all sets of the form $V^{-}$with $V \in \tau$ and all sets of the form $\left(B^{c}\right)^{+}$with $B \in \Delta$. The proximal hit and miss topology $\sigma(\Delta)$ is defined as the topology having as a subbase of all sets of the form $V^{-}$with $V \in \tau_{d}$ and all sets of the form $\left(B^{c}\right)^{++}$with $B \in \Delta$. If we consider the closed balls instead of members of $\Delta$ in the definition of $\sigma(\Delta)$, the resulting topology is called the ball proximal topology, $\tau_{B_{d}}$. The Vietoris topology $\tau_{V}$ is defined as the topology having as a subbase of all sets of the form $V^{-}$with $V \in \tau$ and all sets of the form $W^{+}$with $W \in \tau$. The Wijsman topology $\tau_{W_{d}}$ is the topology having as a subbase all sets of the form;

$$
\{A \in C L(X) \mid d(x, A)<\alpha\} \text { and }\{A \in C L(X) \mid d(x, A)>\alpha\}
$$

for each $x \in X$ and $\alpha>0$, and the proximal topology $\tau_{\Delta_{d}}$ is defined as the topology having as a subbase all sets of the form $V^{-}$with $V \in \tau_{d}$ and all sets of the form $W^{++}$ with $W \in \tau_{d}$. The Fell topology $\tau_{F}$ is defined as the topology having as a subbase all sets of the form $V^{-}$with $V \in \tau$ and all sets of the form $W^{+}$with $W \in \tau$ and $X-W$ is compact, whenever $X$ is a Hausdorff space. For general hyperspace notions we refer to Beer [5].

\section{Definitions and Basic Properties}

The notion of statistical convergence was first introduced by Fast [9] and studied by several authors [8], [11], [13], [20], [24]. This concept is based on the notion of the natural (i.e asiymptotic) density of a subset of positive integers. For a subset $K$ of positive integers $\mathbb{N}$ the natural density of $K$ is given by

$$
\delta(K):=\lim _{n} n^{-1}|\{k \leq n: K \in K\}|
$$

whenever the limit exists, where the vertical bars indicate the number of elements of the enclosed set [10].

In spite of the fact that the notion of statistical convergence was first introduced for real number sequences and then for complex valued sequences, recently several authors have investigated this concept in topological, metric or uniform spaces [6], [7], [18], [26]. 
In a topological space $(X, \tau)$, a sequence $x=\left(x_{k}\right)$ is said to be statistically convergent to $a \in X$ if for any open set $U$ that contains $a$ we have

$$
\delta\left(\left\{k \in \mathbb{N} \mid x_{k} \notin U\right\}\right)=0 .
$$

Note that statistical convergence is stronger than ordinary convergence in an arbitrary topological space and statistical limit is unique whenever the space is a Hausdorff space. Statistical convergence is a topological property and, of course, can be characterized with the elements of the base of the topology instead of the open sets. Therefore the concept of statistical convergence in an arbitrary topological space is nothing new, it is actually a self-evident concept that comes through the structure of that space. For a sequence $x=\left(x_{k}\right)$ in a Hausdorff space $X$, if there exists a subset $K \subset \mathbb{N}$ such that $\delta(K)=1$ and $x$ is convergent to $a \in X$ over $K$ ( $x$ has density convergence property) then it is easy to see that $x$ is statistically convergent to $a$ ([6], [19]). However the converse does not hold in an arbitrary Hausdorff space. It is shown in [6] and [19] independently that if the space is first countable then $x$ has density convergence property if and only if it is statistically convergent. (However there exist examples for which the countability of the base does not hold (see [6]).) The concepts given above can easily be extended to the concepts of $A$-density and $A$-statistical convergence by considering a nonnegative regular summability matrix $A$ ([12], [14], [16], [20]). Moreover if we allow any ideal $I$ instead of the ideal of the $A$-density zero sets we may consider the notion ideal convergence given in [17]. For the sake of simplicity, we keep the concept of statistical convergence in its primary form. Of course similar results in the present paper can be easily considered for $A$-statistical convergence or ideal convergence.

\section{Characterizations}

We first obtain the following characterizations for statistical convergence with respect to the hit and miss, proximal hit and miss, and ball proximal topologies by considering the members of the bases for these topologies.

3.1. Proposition. A sequence $\left(A_{n}\right)_{n \in \mathbb{N}}$ in $C L(X)$ is statistically convergent to $A \in$ $C L(X)$ with respect to the hit and miss topology $\tau(\Delta)$ if and only if both of the following conditions hold:

(i) $\forall V \in \tau \ni A \cap V \neq \emptyset: \delta\left(\left\{k \in \mathbb{N} \mid A_{k} \cap V=\emptyset\right\}\right)=0$

(ii) $\forall B \in \Delta \ni B \cap A=\emptyset: \delta\left(\left\{k \in \mathbb{N} \mid A_{k} \cap B \neq \emptyset\right\}\right)=0$.

Proof. If $\left(A_{n}\right)_{n \in \mathbb{N}}$ is statistically convergent to $A$ in $\tau(\Delta)$, by considering the members of the subbase of $\tau(\Delta)$, it is easy to see that (i) and (ii) hold. Conversely, let $\mathcal{U}$ be an element of the base of $\tau(\Delta)$ containing $A$. Then there exist finite subsets $K, K^{\prime} \subset \mathbb{N}$ such that $\left(V_{k}\right)_{k \in K} \subset \tau,\left(B_{k^{\prime}}\right)_{k^{\prime} \in K^{\prime}} \subset \Delta$ and

$$
\mathcal{U}=\left\{W \in C L(X) \mid V_{k} \cap W \neq \emptyset \text { for each } k \in K \text { and } W \cap \bigcup_{k^{\prime} \in K^{\prime}} B_{k^{\prime}}=\emptyset\right\} .
$$

Since $A \in \mathcal{U}$ we have $A \cap V_{k} \neq \emptyset$ for all $k \in K$ and $A \cap B_{k^{\prime}}=\emptyset$ for all $k^{\prime} \in K^{\prime}$. It follows that

$$
\delta\left(\left\{n \in \mathbb{N} \mid A_{n} \cap V_{k}=\emptyset\right\}\right)=0
$$

for all $k \in K$ and similarly

$$
\delta\left(\left\{n \in \mathbb{N} \mid A_{n} \cap B_{k^{\prime}} \neq \emptyset\right\}\right)=0
$$

for all $k^{\prime} \in K^{\prime}$. Now if $A_{n} \notin \mathcal{U}$ then there exists a $k_{0} \in K$ such that $V_{k_{0}} \cap A_{n}=\emptyset$ or there exists a $k_{0}^{\prime} \in K^{\prime}$ such that $A_{n} \cap B_{k_{0}^{\prime}} \neq \emptyset$. Considering (3.1) and (3.2) we obtain that $\delta\left(\left\{n \in \mathbb{N} \mid A_{n} \notin \mathcal{U}\right\}\right)=0$. Hence the proof is concluded. 
3.2. Proposition. A sequence $\left(A_{n}\right)_{n \in \mathbb{N}}$ in $C L(X)$ is statistically convergent to $A \in$ $C L(X)$ with respect to the proximal hit and miss topology $\sigma(\Delta)$ if and only if both of the following conditions hold:

(i) $\forall V \in \tau \ni A \cap V \neq \emptyset: \delta\left(\left\{k \in \mathbb{N} \mid A_{k} \cap V=\emptyset\right\}\right)=0$

(ii) Whenever $B \in \Delta, \eta>0$ and $S_{\eta}[A] \cap B=\emptyset$, then

$$
\delta\left(\left\{k \in \mathbb{N} \mid S_{\varepsilon}\left[A_{k}\right] \cap B \neq \emptyset \text { whenever } 0<\varepsilon<\eta\right\}\right)=0 .
$$

Proof. It is easily verified that statistical convergence of $\left(A_{n}\right)_{n \in \mathbb{N}}$ to $A$ in $\sigma(\Delta)$ implies (i) and (ii). To prove the opposite direction, let $\mathcal{U}$ be an element of the base of $\sigma(\Delta)$ such that $A \in \mathcal{U}$. Then there exist finite subsets $K, K^{\prime} \subset \mathbb{N}$ such that $\left(V_{k}\right)_{k \in K} \subset \tau_{d}$ and $\left(B_{k}\right)_{k \in K^{\prime}} \subset \Delta$ and

$$
\begin{aligned}
\mathcal{U}= & \left\{W \in C L(X) \mid V_{k} \cap W \neq \emptyset \text { for each } k \in K\right. \text { and } \\
& \left.\exists \eta_{k}>0 \ni S_{\eta_{k}}[W] \subset B_{k}^{c} \text { for each } k \in K^{\prime}\right\} .
\end{aligned}
$$

As $A \in \mathcal{U}$ we have $A \cap V_{k} \neq \emptyset$ for all $k \in K$ and there exists $\eta_{k}>0$ such that $S_{\eta_{k}}[W] \subset B_{k}^{c}$ for all $k \in K^{\prime}$. It follows that, for all $k \in K$ we also have

$$
\delta\left(\left\{n \in \mathbb{N} \mid A_{n} \cap V_{k}=\emptyset\right\}\right)=0
$$

and for all $k \in K^{\prime}$ we have

$$
\delta\left(\left\{n \in \mathbb{N} \mid S_{\varepsilon}[W] \cap B_{k} \neq \emptyset\right\}\right)=0
$$

whenever $0<\varepsilon<\eta=\min _{k \in K^{\prime}} \eta_{k}$. Now if $A_{n} \notin \mathcal{U}$ then there exists a $k_{0} \in K$ such that $V_{k_{0}} \cap A_{n}=\emptyset$ or there exists a $k_{0}^{\prime} \in K^{\prime}$ such that for any $\gamma>0$ we have $S_{\gamma}[W] \cap B_{k_{0}^{\prime}} \neq \emptyset$. In particular, whenever $0<\varepsilon<\eta$ we have $S_{\varepsilon}[W] \cap B_{k} \neq \emptyset$. Hence it follows from (3.3) and (3.4) that $\delta\left(\left\{n \in \mathbb{N} \mid A_{n} \notin \mathcal{U}\right\}\right)=0$.

The proof of the following result goes along the same lines as the previous one, so we omit it.

3.3. Proposition. A sequence $\left(A_{n}\right)_{n \in \mathbb{N}}$ in $C L(X)$ is statistically convergent to $A \in$ $C L(X)$ with respect to the ball proximal topology $\tau_{B_{d}}$ if and only if both of the following conditions hold:

(i) $\forall V \in \tau \ni A \cap V \neq \emptyset: \delta\left(\left\{k \in \mathbb{N} \mid A_{k} \cap V=\emptyset\right\}\right)=0$,

(ii) Whenever $B$ is a closed ball, $\eta>0$ and $S_{\eta}[A] \cap B=\emptyset$, then

$$
\delta\left(\left\{k \in \mathbb{N} \mid S_{\varepsilon}\left[A_{k}\right] \cap B \neq \emptyset \text { whenever } 0<\varepsilon<\eta\right\}\right)=0 .
$$

The Wijsman topology $\tau_{W_{d}}$ for $C L(X)$ can often be represented as a ball proximal hit and miss topology. For instance, this is the case when $X$ is a linear normed space. But in general one can find some examples in literature which shows that the Wijsman topology may not contain the ball proximal topology (see e.g [5]). In the case of ordinary convergence, we know that a sequence $\left(A_{n}\right)_{n \in \mathbb{N}}$ in $C L(X)$ is convergent to $A \in C L(X)$ with respect to $\tau_{W_{d}}$ if and only if the following conditions hold:

(i) Whenever $A$ meets a nonempty open subset $V$ of $X$, then there exists $n_{0} \in \mathbb{N}$ such that $A_{n} \cap V \neq \emptyset$ for all $n \geq n_{0}$.

(ii) Whenever $0<\varepsilon<\eta$ and $S_{\eta}[x] \cap A=\emptyset$, then there exists $n_{0} \in \mathbb{N}$ such that $A_{n} \cap S_{\varepsilon}[x]=\emptyset$ for all $n \geq n_{0} .[5]$.

With the help of this fact the following result shows how we have to characterize statistical convergence with respect to the Wijsman topology in general.

3.4. Theorem. A sequence $\left(A_{n}\right)_{n \in \mathbb{N}}$ in $C L(X)$ is statistically convergent to $A \in C L(X)$ with respect to $\tau_{W_{d}}$ if and only if both of the following conditions hold: 
(i) $\forall V \in \tau_{d} \ni A \cap V \neq \emptyset: \delta\left(\left\{k \in \mathbb{N} \mid A_{k} \cap V=\emptyset\right\}\right)=0$,

(ii) Whenever $0<\varepsilon<\eta, x \in X$ and $S_{\eta}[x] \cap A=\emptyset$, then

$$
\delta\left(\left\{k \in \mathbb{N} \mid S_{\varepsilon}[x] \cap A_{k} \neq \emptyset\right\}\right)=0 .
$$

Proof. Assume that $\left(A_{n}\right)$ is statistically convergent to $A$ in $\left(C L(X), \tau_{W_{d}}\right)$ and $V \in \tau_{d}$ such that $A \cap V \neq \emptyset$. Then there exists $\mu>0$ and $x_{0} \in A \cap V$ such that $S_{\mu}\left[x_{0}\right] \subset V$. Now if we define

$$
\mathcal{W}:=\left\{U \in C L(X) \mid d\left(x_{0}, U\right)<\mu\right\}
$$

then clearly $\mathcal{W} \in \tau_{W_{d}}$ and $A \in \mathcal{W}$. It follows that $\delta\left(\left\{n \in \mathbb{N} \mid A_{n} \notin \mathcal{W}\right\}\right)=0$. Now, as $S_{\mu}\left[x_{0}\right] \cap A_{n}=\emptyset$ we have

$$
\left\{n \in \mathbb{N} \mid A_{n} \cap V=\emptyset\right\} \subset\left\{n \in \mathbb{N} \mid A_{n} \notin \mathcal{W}\right\}
$$

which implies that $\delta\left(\left\{n \in \mathbb{N} \mid A_{n} \cap V=\emptyset\right\}\right)=0$. On the other hand, whenever $0<\varepsilon<\eta$ and $S_{\eta}[x] \cap A=\emptyset$, consider the open set in $\tau_{W_{d}}$ defined by

$$
\mathcal{W}:=\{U \in C L(X) \mid d(x, U)>\varepsilon\} .
$$

From the fact that

$$
\left\{n \in \mathbb{N} \mid S_{\varepsilon}[x] \cap A_{n} \neq \emptyset\right\} \subset\left\{n \in \mathbb{N} \mid A_{n} \notin \mathcal{W}\right\}
$$

we have $\delta\left(\left\{k \in \mathbb{N} \mid S_{\varepsilon}[x] \cap A_{k} \neq \emptyset\right\}\right)=0$.

Conversely assume that $(i)$ and $(i i)$ hold and consider the following sets

$$
\mathcal{U}_{A}=\left\{V \in \tau_{d} \mid A \cap V \neq \emptyset\right\} \text { and } \mathcal{V}_{A}=\left\{S_{\eta}[x] \mid S_{\eta}[x] \cap A=\emptyset\right\}
$$

where $x \notin A$. Observe that for any $V \in \mathcal{U}_{A}$ and $S_{\eta}[x] \in \mathcal{V}_{A}$ we have $\delta\left(K_{V, \varepsilon}\right)=1$ for each positive $\varepsilon$ less than $\eta$ where $K_{V, \varepsilon}=K_{V}^{c} \cap K_{\varepsilon}^{c}, K_{V}=\left\{n \in \mathbb{N} \mid A_{n} \cap V=\emptyset\right\}$ and $K_{\varepsilon}=\left\{n \in \mathbb{N} \mid S_{\varepsilon}[x] \cap A \neq \emptyset\right\}$. Furthermore, since $A_{n} \cap V \neq \emptyset$ and $S_{\eta}[x] \cap A \neq \emptyset$ for any $n \in K_{V, \varepsilon}$ we obtain that $\left(A_{n}\right)$ converges to $A$ over $K_{V, \varepsilon}$ in $\tau_{W_{d}}$. Hence $\left(A_{n}\right)$ is statistically convergent to $A$ in Wijsman topology.

The following lemma characterizes the statistical convergence in weak topologies.

3.5. Lemma. For a nonempty set $X$ and a family of Hausdorff topological spaces $\left\{\left(X_{i}, \tau_{i}\right)\right\}_{i \in I}$, consider the weak topology $\tau_{\mathfrak{R}}$ on $X$ determined by

$$
\mathfrak{R}=\left\{f_{i}: X \longrightarrow X_{i}\right\}_{i \in I} \text {. }
$$

Then, for an arbitrary sequence $x$ in $X$ and $x_{0} \in X$, the following are equivalent:

(i) $s t_{\tau_{\mathfrak{R}}}-\lim x=x_{0}$,

(ii) $\forall i \in I: s t_{\tau_{\mathrm{i}}} \lim f_{i}(x)=f_{i}\left(x_{0}\right)$.

Proof. Since $f_{i}$ is continuous for all $i \in I$ it is not difficult to see that (i) implies (ii). Conversely assume that $s t_{\tau_{\mathrm{i}}}-\lim f_{i}(x)=f_{i}\left(x_{0}\right)$ for all $i \in I$. Let $B$ be an element of the base of $\tau_{\mathfrak{R}}$ such that $x_{0} \in B$. Then there exists $i_{1}, i_{2}, \ldots, i_{m} \in I$ such that $B=\bigcap_{p=1}^{m} f_{i_{p}}^{-1}\left(U_{i_{p}}\right)$ where $U_{i_{p}} \in \tau_{i}$ for all $p=1,2, \ldots, m$. It follows that $f_{i_{p}}\left(x_{0}\right) \in U_{i_{p}}$ for all $p=1,2, \ldots, m$. Then one can obtain that for each $p=1,2, \ldots, m$

$$
\delta\left(\left\{n \in \mathbb{N} \mid f_{i_{p}}\left(x_{n}\right) \notin U_{i_{p}}\right\}\right)=0 .
$$

Now if $x_{n} \notin B$, then there exists $p_{o} \in\{1,2, \ldots, m\}$ such that $x_{n} \notin f_{i_{p_{0}}}^{-1}\left(U_{i_{p_{0}}}\right)$. Hence by (3.5) we have $\delta\left(\left\{n \in \mathbb{N} \mid x_{n} \notin B\right\}\right)=0$ which completes the proof. 
3.6. Remark. From the fact that the Wijsman topology is the weak topology generated by the family of the distance functions $\left\{d_{x}: C L(X) \longrightarrow \mathbb{R}_{+}\right\}_{x \in X}$ where $d_{x}(A):=$ $d(x, A)$, statistical convergence of a sequence $\left(A_{n}\right)_{n \in \mathbb{N}}$ to $A$ in $\tau_{W_{d}}$ is actually means that, for each $x \in X$, we have $s t$ - $\lim _{n \rightarrow \infty} d_{x}\left(A_{n}\right)=d_{x}(A)$.

Also one can look differently by considering the fact that $B \longleftrightarrow d_{B}$ is an embedding of $\left(C L(X), \tau_{W_{d}}\right)$ into the space of continuous functions $C(X, \mathbb{R})$ equipped with the topology of pointwise convergence. Using the fact that statistical convergence is a topological property, it follows that statistical convergence of $\left(A_{n}\right)_{n \in \mathbb{N}}$ in Wijsman topology is determined by the statistical convergence of $\left(d_{A_{n}}\right)_{n \in \mathbb{N}}$, in $C(X, \mathbb{R})$ equipped with the topology of pointwise convergence. In addition, the admissibility of the Wisjman topology provides that all the topological results obtained for the hyperspace case are valid for the ordinary case.

3.7. Proposition. A sequence $\left(A_{n}\right)_{n \in \mathbb{N}}$ in $C L(X)$ is statistically convergent to $A \in$ $C L(X)$ with respect to the Vietoris topology $\tau_{V}$ if and only if both of the following conditions are met:

(i) $\forall V \in \tau \ni A \cap V \neq \emptyset: \delta\left(\left\{k \in \mathbb{N} \mid A_{k} \cap V=\emptyset\right\}\right)=0$

(ii) $\forall V \in \tau \ni A \subset V: \delta\left(\left\{k \in \mathbb{N} \mid A_{k} \nsubseteq V\right\}\right)=0$.

Similar to the Wisjman topology, Vietoris topology is actually the weak topology determined by the family of distance functionals $\left\{d_{x} \mid x \in X, d \in \mathfrak{D}\right\}$ whenever $\mathfrak{D}$ is the set of compatible metrics for $X$. Then it follows from Lemma 1 that statistical convergence of a sequence $\left(A_{n}\right)$ to $A$ in $C L(X)$ is actually means that, for each $x \in X$ and for each $d \in \mathfrak{D}$, we have $s t$ - $\lim _{n \rightarrow \infty} d_{x}\left(A_{n}\right)=d_{x}(A)$.

The proximal topology on the closed sets of a metric space $(X, d)$ is the weak topology determined by the family of distance functions $\left\{q_{x} \mid x \in X, q \in \mathfrak{D}_{d}\right\}$ whenever $\mathfrak{D}_{d}$ denotes the set of compatible metrics for $d$. Then it follows from Lemma 1 that the statistical convergence in proximal topology can be determined by the family of such kind of distance functions. Therefore statistical convergence of a sequence $\left(A_{n}\right)_{n \in \mathbb{N}}$ to $A$ in $C L(X)$ is actually means that, for each $x \in X$ and $q \in \mathfrak{D}_{d}$, we have st- $\lim _{n \rightarrow \infty} q_{x}\left(A_{n}\right)=q_{x}(A)$. In addition; using the fact that $\tau_{\Delta_{d}}$ is also the weak topology determined by the family of gap functions $\left\{D_{d}(B,) \mid. B \in C L(X)\right\}$ one can observe that $s t_{\tau_{\Delta_{d}}}-\lim _{n \rightarrow \infty} A_{n}=A$ means that $s t-\lim _{n \rightarrow \infty} D_{d}\left(B, A_{n}\right)=D_{d}(B, A)$ for each $B \in C L(X)$.

The Attouch-Wets topology on $C L(X)$ is the topology that $C L(X)$ inherits from $C(X, \mathbb{R})$, equipped with the topology $\tau_{u c b}$ of uniform convergence on bounded subsets of $X$, under the identification $B \longleftrightarrow d_{B}$. The following characterization is a consequence of this fact.

3.8. Proposition. A sequence $\left(A_{n}\right)_{n \in \mathbb{N}}$ of bounded sets in $C L(X)$ is statistically convergent to a bounded set $A$ in $C L(X)$ with respect to the Attouch-Wets topology $\tau_{A W_{d}}$ if and only if for every $\varepsilon>0$,

$$
\delta\left(\left\{k: d_{u c b}\left(d_{A_{k}}, d_{A}\right) \geq \varepsilon\right\}\right)=0
$$

whenever

$$
d_{u c b}(f, g)=\sum_{n} 2^{-n} \min \left\{1, \sup _{d\left(x, x_{0}\right)<n}|f(x)-g(x)|\right\} \text { for all } f, g \in C(X, \mathbb{R}) .
$$

A similar fact also holds for the Hausdorff metric topology $\tau_{H_{d}}$ on $C L(X)$ since it is the topology that $C L(X)$ inherits from $\left(C(X, \mathbb{R}), \tau_{u c}\right)$ under the identification $B \longleftrightarrow d_{B}$. Therefore, $\left(A_{n}\right)_{n \in \mathbb{N}}$ in $C L(X)$ is statistically convergent to $A \in C L(X)$ with respect to 
$\tau_{H_{d}}$ means that:

$$
\forall \varepsilon>0: \delta\left(\left\{k: \sup _{x \in X}\left|d\left(x, A_{k}\right)-d(x, A)\right|>\varepsilon\right\}\right)=0 .
$$

In addition, due to the fact that $\tau_{H_{d}}$ is the weak topology on $C L(X)$ such that for each $B \in C L(X)$ both

$$
A \longrightarrow D_{d}(B, A) \text { and } A \longrightarrow e_{d}(B, A)
$$

are continuous, $\left(A_{n}\right)_{n \in \mathbb{N}}$ in $C L(X)$ is statistically convergent to $A \in C L(X)$ with respect to $\tau_{H_{d}}$ means that:

$$
s t-\lim _{n \rightarrow \infty} D_{d}\left(B, A_{n}\right)=D_{d}(B, A)
$$

and

$$
s t-\lim _{n \rightarrow \infty} e_{d}\left(B, A_{n}\right)=e_{d}(B, A)
$$

for each $B \in C L(X)$.

The proof of the following result is a consequence of the definition of Fell topology.

3.9. Proposition. If $X$ is a Hausdorff space, then a sequence $\left(A_{n}\right)_{n \in \mathbb{N}}$ in $C L(X)$ is statistically convergent to $A \in C L(X)$ with respect to the Fell topology $\tau_{F}$ if and only if both of the following conditions are met:

(i) $\forall V \in \tau \ni A \cap V \neq \emptyset: \delta\left(\left\{k \in \mathbb{N} \mid A_{k} \cap V=\emptyset\right\}\right)=0$

(ii) $\forall W \in \tau \ni A \subset W$ and $X \backslash W$ compact $: \delta\left(\left\{k \in \mathbb{N} \mid A_{k} \nsubseteq W\right\}\right)=0$.

In the realm of function spaces, the characterization of statistical convergence for graph topology with base $\left\{W^{+} \mid W\right.$ open in $\left.X \times Y\right\}$ which is much stronger than $\tau_{\Delta_{d}}$ is straightforward. Clearly, a sequence $\left(f_{n}\right)_{n \in \mathbb{N}}$ in $C(X, Y)$ is said to be statistically convergent to $f \in C(X, Y)$ with respect to the graph topology if and only if for each open set $W$ in $X \times Y$ containing $f$, we have $\delta\left(\left\{k \in \mathbb{N} \mid f_{k} \nsubseteq W\right\}\right)=0$.

\section{References}

[1] Attouch, H., Lucchetti, R. and Wets, R. The topology of the $\rho$-Hausdorff distance, Ann. Mat. Pura. Appl. 160, 303-320, 1991.

[2] Beer, G. Metric spaces with nice closed balls and distance function for closed sets, Bull. Austral. Math. Soc. 35, 81-96, 1978.

[3] Beer, G. On Mosco convergence of convex sets, Bull. Austral. Math. Soc. 95, 737-739, 1988.

[4] Beer, G. An embedding theorem for the Fell topology, Michigan Math. J. 35, 3-9, 1988.

[5] Beer, G. Topologies on closed and convex sets, Math. App. 268, Kluwer Academic Publishers Group, Dordrecht, 1993.

[6] Cakalli, H. and Khan, M. K. Summability in topological spaces, Appl. Math. Lett. 24 (3), 348-352, 2011.

[7] Cakalli, H. Lacunary statistical convergence in topological groups, Indian J. Pure Appl. Math. 26 (2), 113-119, 1995.

[8] Connor, J. S. The statistical and strong p-Cesàro convergence of sequences, Analysis 8, $47-63,1988$.

[9] Fast, H. Sur la convergence statistique, Colloq. Math. 2, 241-244, 1951.

[10] Freedman, A.R. and Sember, J.J. Densities and summability, Pacific J. Math. 95, 293-305, 1981.

[11] Fridy, J.A. On statistical convergence, Analysis 5, 301-313, 1985.

[12] Fridy, J.A. and Miller H. I. A matrix characterization of statistical convergence, Analysis 11, 59-66, 1991.

[13] Fridy, J.A. and Orhan, C. Statistical limit superior and limit inferior, Proc. Amer. Math. Soc. 125 (12), 3625-3631, 1997. 
[14] Khan, M.K. and Orhan, C. Matrix characterization of A-statistical convergence, J. Math. Anal. Appl. 335, 406-417, 2007.

[15] Kişi, Ö. and Nuray, F. New convergence definitions for sequences of sets, Abst. Appl. Anal. Volume 2013, Article ID 852796.

[16] Kolk, E. Matrix summability of statistically convergent sequences, Analysis 13 (1-2), 77-83, 1993.

[17] Kostyrko, P., Macaj, M. and Šalát, T. I-convergence, Real Anal. Exchange 26 (2), 669-686, 2000.

[18] Maddox, I.J. Statistical convergence in a locally convex space, Math. Proc. Cambridge Philos. Soc. 104 (1), 141-145, 1988

[19] Maio, G. D. and Kočinac, L.D.R. Statistical convergence in topology, Topology Appl. 156, $28-45,2008$.

[20] Miller, H.I. A measure theoretical subsequence characterization of statistical convergence. Trans. Amer. Math. Soc. 347 (5), 1811-1819, 1995.

[21] Mosco, U. Convergence of convex sets and of solutions of variational inequalities, Advances in Math. 3 (4), 510-585, 1969.

[22] Nuray, F. and Rhoades, B.E. Statistical convergence of sequences of sets, Fasc. Math. 49, 87-99, 2012.

[23] Pancaroğlu, N. and Nuray, F. Invariant statistical convergence of sequences of sets with respect to a modulus function, Abst. Appl. Anal. Volume 2014, Article ID 818020.

[24] Šalát, T. On statistically convergent sequences of real numbers, Math. Slovaca, 30 (2), 139-150, 1980.

[25] Savaş, E. On I-lacunary statistical convergence of order $\alpha$ for sequences of sets, Filomat, $29(6), 2015$.

[26] Unver, M., Khan, M. K. and Orhan, C. A-distributional summability in topological spaces, Positivity, 18 (1), 131-145, 2014.

[27] Wets, R. Convergence of convex functions, variational inequalities, and convex optimization problems, Variational inequalities and complementarity problems 375-403, 1980.

[28] Wijsman, R.A. Convergence of sequences of convex sets, cones and functions, II. Trans. Amer. Math. Soc. 123, 32-45, 1966. 